\title{
Entosis, a key player in cancer cell competition
}

\author{
Cell Research (2014) 24:1280-1281. doi:10.1038/cr.2014.133; published online 24 October 2014
}

Cell-in-cell structures, also referred to as 'entosis', are frequently found in human malignancies, although their prognostic impact remains to be defined. Two articles recently published in Cell Research report the stimulation of entosis by one prominent oncogene, Kras, as well as by one class of tumor suppressors, namely epithelial cadherins $E$ and $P$, illustrating the complex regulation of this biological process.

A number of different terms have been used to describe live cell engulfments giving rise to cell-in-cell structures (CICS): entosis, emperipolesis, cannibalism and phagocytosis. Heterotypic live cell engulfment usually involves the ingestion of leukocytes by non-leukocytes (such as epithelial cells or fibroblasts). Homotypic live cell engulfment (among cells of the same type) mostly occurs in cancers, probably reflecting major alterations in cellular physiology that are associated with oncogenesis and tumor progression.

CICS can be visualized by conventional hematoxylin-eosin staining and have been described to occur in many different human cancers [1]. CICS produced as the result of entosis exhibit $\beta$-catenin localization patterns that are indicative of a cell junction-mediated mechanism of engulfment, and this polarized distribution of $\beta$-catenin can be taken advantage of to visualize CICS in vivo, in tumors [1]. However, the prognostic impact of CICS is highly context-dependent. Thus, CICS are particularly frequent in high-grade, aggressive breast cancer with dismal prognosis [2]. CICS are only found in castration-resistant, not in androgen- dependent, prostate cancer and hence correlate with poor prognosis in this particular malignancy [3]. In contrast, in pancreas adenocarcinomas, high levels of CICS correlate with a lower incidence of metastases [4]. These findings point to a complex role of CICS in cancer biology.

Two papers by Sun et al. [5, 6] recently published in Cell Research characterized one particular mechanism of homotypic live cell engulfment termed entosis. The first paper of this series [5] provides evidence that one of the most prominent oncogenes, activated Kras, can stimulate entosis, while the second paper [6] demonstrates that a prominent tumor suppressor, epithelial cadherin (E-cadherin), can increase entosis as well.

Cancers are highly complex mixtures of cells in which the malignant population is genetically and epigenetically heterogeneous, reflecting a history of clonal selection. One particular type of competition among distinct cells may consist in the engulfment of one cell (the 'loser') by another (the 'winner'), as demonstrated by Sun et al. in several cell culture models, as well as in human cancers that were xenografted into immunodeficient mice [5]. Importantly, co-culture of non-transformed cells with their malignant counterparts systematically leads to engulfment of the former by the latter, suggesting that oncogenic transformation is coupled to the "winner' status [5]. Indeed, competition by entosis leads to the physical elimination of the 'loser' cells, which usually succumb to non-apoptotic cell death as soon as the phagosome enveloping the engulfed cell is decorated with LC3 and then fuses with lysosomes $[1,7]$. What is then the difference between 'loser' and 'winner' cells? Sun et al. [5] propose that one cardinal feature of 'winners' is a high degree of mechanic deformability, as demonstrated by biophysical experiments and computer simulations. This is a highly provocative finding because human tumors are known to be more mechanically heterogeneous than normal tissues and that tumor progression is increased with an elevated mechanic deformability of the cancer cells. This reduction in cell stiffness may hence not only increase the metastatic potential of tumor cells [8], but may also reflect an increased entotic activity [5].

Transfection-enforced expression of active KrasV12 was sufficient to confer winner status onto non-tumorigenic cells, correlating with an increase in mechanic deformability [5]. This effect of KrasV12 relied on Rac1, as demonstrated by the facts that knockdown of Rac1 suppressed the 'winner' status conferred by KrasV12, expression of constitutively active Rac1 induced a 'winner' phenotype and dominantnegative Rac1N17 imparted a 'loser' status [5]. However, at this point it remains to be explored whether other pathways downstream of Kras such as the phosphatidylinositide 3-kinases $(\mathrm{PI} 3 \mathrm{~K}) /$ protein kinase $\mathrm{B}$ (PKB, best known as AKT)/mechanistic target of rapamycin (mTOR) pathway may contribute to 'winner' status. Inhibition of mTOR interferes with degradation of engulfed cells [9], suggesting that activation of the PI3K/AKT/mTOR axis might favor the manifestation of the 'winner' phenotype as well. Similarly, it 
A

\section{Entosis as a tumor accelerator}

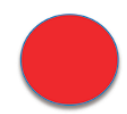

Malignant cell
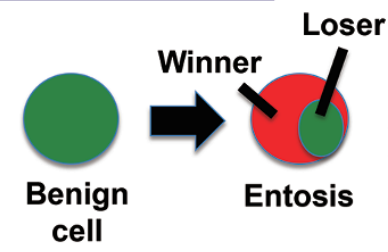

,

Entosis in tumor suppression

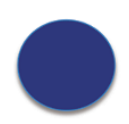

Cell A
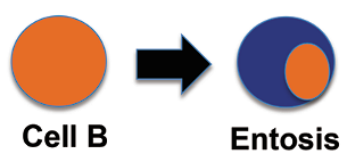

Figure $1 \mathrm{~A}$ dual role for entosis in cancer. (A) Entosis as a pro-tumorigenic process. (B) Entosis as a tumor-suppressive mechanism.

remains an open question as to whether other oncogenes than Kras may regulate entosis as well.

Breast cancers cells engineered to express epithelal E- or P-cadherins (but not mesenchymal-type cadherins, such as N-cadherin and cadherin-11) re-establish epithelial junctions and engulf and kill non-transfected parental cells in transformed growth assays [6]. The induction of entosis by epithelial E- or P-cadherins is associated to the polarized distribution of RhoA and contractile actomyosin dependent on the p190A Rho-GTPase-Activating Protein (p190A RhoGAP) that is recruited to epithelial junctions [6]. Inhibition of RhoA by overexpression of RhoAN19 or p190A RhoGAP was sufficient to impart winner status to cells mixed with controls, whereas overexpression of RhoA, ROCKI, or ROCKII had the opposite effect and hence created 'loser' cells [5]. It has been known that RhoGTPase and Rho-kinase are not required in engulfing cells but are required in internalizing cells [1], underscoring the idea that 'loser' cells are not just passive 'victims' of a cannibalistic attack but somehow contribute to their fatal fate. The 'loser' status was accompanied by

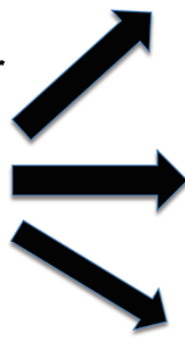

Malignant cells outcompete benign cells

Winners retrieve nutrients from losers

Mitotic defects facilitate genomic instability

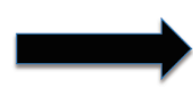

Repeated entosis reduces clonogenic potential in mixed culture experiments [5]. On the other hand, stimulation of entosis by re-expression of epithelal E- or $\mathrm{P}$-cadherins reduced the clonogenic potential of breast cancer cells. In this context, Y27632 facilitated tumor cell growth in vitro [6]. These observations underscore the need of exploring the detailed mechanisms through which entosis may repress or favor oncogenesis and tumor progression.

\section{Guido Kroemer ${ }^{1,2,3,4}$, Jean-Luc Perfettini ${ }^{5,6,7,8}$}

${ }^{1}$ Equipe 11 labellisée par la Ligue Nationale contre le Cancer, Centre de Recherche des Cordeliers, INSERM U1138, F-75006 Paris, France; ${ }^{2}$ Université Paris Descartes, Sorbonne Paris Cité, F-75005 Paris, France; ${ }^{3}$ Metabolomics and Cell Biology Platforms, Gustave Roussy, F-94805 Villejuif, France; ${ }^{4}$ Pôle de Biologie, Hôpital Européen Georges Pompidou, AP-HP, F-75015 Paris, France; ${ }^{5}$ Cell death and Aging team, Gustave Roussy, F-94805 Villejuif, France; ${ }^{6}$ Laboratory of Molecular Radiotherapy, INSERM U1030, Gustave Roussy, F-94805 Villejuif, France; 'Gustave Roussy, F-94805 Villejuif, France; ${ }^{8}$ Université Paris Sud - Paris 11, F-94805 Villejuif, France

Correspondence: Guido Kroemer

E-mail: kroemer@orange.fr

\section{References}

' [6]. RhoA, ROCKI/II, MLC2, actin and myosins all accumulated at particularly high levels in 'losers' at the cell cortex oriented away from cell-cell adhesions [6].

The aforementioned data support a dual implication of entosis in carcinogenesis (Figure 1). On one hand, entosis carried out by 'winner' cells may constitute a competitive advantage of aggressive tumor cells, perhaps allowing the 'winners' to retrieve amino acids and other building blocks for anabolic reaction from their cannibalistic activity [9] or increasing their genomic instability subsequent to mitotic aberrations $[2,10]$. In this context, pharmacological suppression of entosis by Y27632, a ROCKI/II inhibitor, abolished the competitive advantage of transformed cells over their non-transformed siblings
1 Overholtzer M, Mailleux AA, Mouneimne G, et al. Cell 2007; 131:966-979.

2 Krajcovic M, Johnson NB, Sun Q, et al. Nat Cell Biol 2011; 13:324-330.

3 Wen S, Shang Z, Zhu S, et al. Prostate 2013; 73:1306-1315.

4 Cano CE, Sandí MJ, Hamidi T, et al. EMBO Mol Med 2012; 4:964-979.

5 Sun Q, Luo T, Ren Y, et al. Cell Res 2014; 24:1299-1310.

6 Sun Q, Cibas ES, Huang H, et al. Cell Res 2014; 24:1288-1298.

7 Florey O, Kim SE, Sandoval CP, et al. Nat Cell Biol 2011; 13:1335-1343.

$8 \mathrm{Xu}$ W, Mezencev R, Kim B, et al. PLoS One 2012; 7:e46609.

9 Krajcovic M, Krishna S, Akkari L, et al. Mol Biol Cell 2013; 24:3736-3745.

10 Vitale I, Galluzzi L, Senovilla L, et al. Cell Death Differ 2011; 18:1403-1413. 